\title{
Discrimination reversal learning in naive stump-tailed monkeys as a function of number of acquisition trials'
}

\author{
William N. Boyer, OKLAHOMA STATE UNIVERSITY \\ Henry A. Cross, teXas teChNological COLLEG
}

\begin{abstract}
An object-discrimination group and a position-discrimination group each received four 10-trial and four 20-trial acquisition problems. Following each problem all Ss were given 20 reversal training trials. The results demonstrated that reversal performance following 10 trials was superior to performance after 20 trials. There was no significant difference in reversal performance attributable to training method.

\section{Problem}

Using squirrel monkeys in a two-object discrimination task, Cross \& Brown (1965), and Cross, Fickling, Carpenter \& Brown (1964) observed that the greater the number of prereversal discrimination trials, the poorer the reversal performance. The present study was an attempt to directly compare the effect of objectand position-discrimination training procedures upon reversal performance. In addition, the design permitted a further evaluation of the influence of initial trials on reversal behavior with another and little tested monkey.
\end{abstract}

\section{Method}

The Ss were four male and four female mature stumptailed monkeys, M a caca specios a, from the colony at the University of Wisconsin Primate Research Center. All Ss were tested in a standard WGTA, utilizing multidimensional objects maintained at the laboratory. Adaptation consisted in training Ss to displace a gray block covering the center foodwell for a raisin reward.

The Ss were randomly assigned to two groups of four each. These groups constituted the object-and positiondiscrimination groups respectively. Problems for both groups involved simultaneous discrimination between two dissimilar stimulus objects. For the object-discrimination group the positive stimulus appeared randomly on the left and right side, the number of times the positive object appeared on each side being balanced for each 10 trials. For the position-discrimination group, the cue-correlated procedure (D'Amato \& Schiff, 1964) was employed and the positive stimulus appeared on the same side thoughout the entire problem. The rewarded side for a particular problem in the positiondiscrimination condition was randomly determined with the restriction that there be an equal number of left and right problems.

The Ss within each group received four 10-trial and four 20-trial acquisition problems. The order of problems was randomly determined for each $\mathrm{S}$ with the condition that two 10-trial and two 20-trial problems appear within each block of four problems. Immediately upon completion of each acquisition problem all Ss were given 20 reversal trials. Reversal training for each group was identical in procedure to initial discrimination learning except that the previously positive stimulus object was now negative. All Ss received one problem per day over an eight-day period. The non-correction method was employed with raisin as a reward.

\section{Results}

The overall percentage of correct responses during acquisition learning for the 10- and 20-trial discrimination problems was $66 \%$ and $70 \%$ respectively. For the object- and position-discrimination procedures, the percentage of correct choices was $65 \%$ and $72 \%$. Percentage scores were subjected to arcsine transformation and an analysis of variance was performed. The analysis showed that initial discrimination learning was not influenced by length of problem $(F=.38 ; \mathrm{df}=1 / 6)$. Similarly, the difference between the object and position learning procedures was not significant $(F=1.85$; $d f=$ $1 / 6)$.

Reversal learning performance was analyzed over varying numbers of reversal trials. A separate analysis of variance was conducted on these data after the percentage scores were converted into arcsine transformation scores. One significant effect was obtained and this appeared in the overall 20-trial analysis (see Table 1). Reversal performance was poorer following 20 acquisition trials than after 10 such trials $(F=36.48$; $\mathrm{df}=1 / 6 ; \mathrm{p}<.01)$. There was no significant difference between the position- and object-discrimination training methods $(F=.002 ; d f=1 / 6)$. No other effects or interactions were significant.

\section{Diseussion}

The present results show that as animals receive more initial trials on a discrimination problem, their reversal

Table 1. Mean Transformed Reversal Scores for 10and 20-Trial Object- and Position-Discrimination Problems

Type of Problem

Object-Discrimination

\begin{tabular}{cc} 
10-Trial & $20-$ Trial \\
50.95 & 45.72 \\
49.86 & 47.12 \\
& \\
\hline
\end{tabular}

Totals

50.40

46.42 
performance decreases. These data confirm those reported by Cross \& Brown (1965), and Cross et al (1964), and extend the generality of the finding to include stump-tailed monkeys and two quite different acquisition procedures.

The finding that method of training revealed no differential effect either upon initial learning or upon reversal performance was unexpected. From a cursory consideration of the two different methods, it would appear that the position-discrimination procedure employed would offer two possible cues, those of object and position, while the object-discrimination procedure affords only one cue. The failure to find differences between training methods may be related to the fact that Ss had not had sufficient experience in solving discrimination problems to utilize more than one cue. In any event,

\section{Comment}

In a recent article (The effect of false-information feedback upon psychophysical judgments. Psychon. Sci., 1964, 1, 317-318), Kinchla and Atkinson raise the question of "whether the introduction of false information on no-signal trials gives rise to points that fall on the ROC curve defined by the experimental conditions involving no misinformation." Regrettably, the authors fail to describe the form of their theoretical ROC curve and present no ROC curves "defined by" the two points obtained under conditions of no misinformation. As a consequence I fail to see how Kinchla and Atkinson could hope to establish whether or not misinformation produces points either on or off such an ROC curve. Nevertheless we are told that, on the basis of inspection, "the four points fall on the same ROC curve" and that "a simple linear ROC curve would provide a reasonably good account of almost every S's data."

Now it seems clear that if Kinchla and Atkinson meant to predict linear ROC curves for yes-no data-a dubious proposition in the light of Atkinson's theory, as well as others-they should have drawn a line through the two points obtained for $\lambda=0$ and looked to see whether the two points obtained under misinformation might be said to lie upon it. If we do this, we find that they lie beneath it ( 9 of $14 \mathrm{Ss}$ for $\gamma=.25, \lambda=.33$, and 12 of 14 Ss for $\gamma=.50, \lambda=.50)$. If, on the other hand, Kinchla and Atkinson meant to predict nonlinear ROC curves, then their claim that a linear function well describes their data implies unequal detectability for their four experimental conditions. In either case Kinchla and Atkinson should have been led to conclude that misinformation and correct information give rise to points which do not lie on the same ROC curve. As a matter of fact, however, most listeners' data is better fitted by a curvilinear ROC curve of the Tanner-Swets-Green variety than by a linear function. (The apparent linearity of even the averaged-data curve in Kinchla and these data demonstrated that regardless of training method, Ss consistently performed less well in reversal as the number of acquisition trials was increased.

\section{References}

CROSS, H. A., \& BROWN, L. T. Discrimination reversal learning in squirrel monkeys as a function of number of acquisition trials and prereversal experience. J. comp. physiol. Psychol., 1965, in press.

CROSS, H. A., FICKLING, R. M., CARPENTER, J. B., \& BROWN, L. T. Discrimination reversal performance in squirrel monkeys as a function of prereversal experience and overlearning. Psychon. Sci., 1964, 1, 353-354.

D'AMATO, M. R., \& SCHIFF, D. Further studies of overlearning and position reversal learning. Psychol. Rep., 1964, 14, 380-382. Note

1. The authors are grateful to Dr. Harry F. Harlow of the Regional Primate Center, Madison, Wisconsin for generously making available the subjects and equipment employed in this investigation.

Atkinson's Fig. 1 is less convincing if we replot the data points on a less compressed scale.) Moreover, if we take the four average coordinate pairs, convert their proportions to z-scores, and replot the points on normal-normal, rather than on linear, coordinate paper, we find that they are fitted nearly perfectly by a straight line. On the strength of this evidence it may be reasonable to conclude that a single ROC curve encompasses performance obtained under conditions of correct information and misinformation. I am afraid that the analysis that incorrectly led Kinchla and Atkinson to the same conclusion serves only to mislead their readers.

The authors also conclude that "false-information feedback increases both the probability of a hit and of a false alarm, and at the same time reduces the overall probability of a correct response." This conveys the erroneous impression that $\mathrm{p}(\mathrm{C})$ may vary independently of $p\left(A_{1}\right)$. In fact $p(C)$ varies according to $p\left(A_{1}\right)$, the detectability of the signal, and the a priori signal probability. If a listener operates at the point on his ROC curve where $p(C)$ is a maximum-a point Kinchla and Atkinson's listeners seem to approach when they are not misinformed-then any change in $\mathrm{p}\left(\mathrm{A}_{1}\right)$, upwards or downwards, inexorably leads to fewer correct judgments.

Finally, Kinchla and Atkinson state that "the ratio of signal energy to noise power in a one cycle band $\left[\mathrm{E} / \mathrm{N}_{\mathrm{O}}\right] "$ was held constant at 2.9 throughout their experiment. The level of detectability indicated by the ROC curves they present, however, is considerably and inexplicably better than what one expects for $\mathrm{E} / \mathrm{N}_{\mathrm{O}}=\mathbf{2 . 9}$. About three times this value of $\mathrm{E} / \mathrm{N}_{\mathrm{O}}$ is usually required to achieve the level of detectability they have obtained.

Arthur I. Schulman Lincoln Laboratory* Massachusetts Institute of Technology (*Operated with support from the U.S. Air Force.)

(See page 132 for reply by $\mathrm{R}$. A. Kinchla and R. C. Atkinson.) 